\title{
AMENDMENTS
}

\section{Author Correction: Excitons in strain-induced one-dimensional moiré potentials at transition metal dichalcogenide heterojunctions}

Yusong Bai (D), Lin Zhou, Jue Wang (D), Wenjing Wu (D), Leo J. McGilly, Dorri Halbertal, Chiu Fan Bowen Lo, Fang Liu, Jenny Ardelean, Pasqual Rivera (D), Nathan R. Finney, Xu-Chen Yang (D), D. N. Basov (D), Wang Yao (D), Xiaodong Xu (D), James Hone (iD, Abhay N. Pasupathy (D) and X.-Y. Zhu (D)

Correction to: Nature Materials https://doi.org/10.1038/s41563-020-0730-8, published online 13 July 2020.

In the version of this Article originally published, the first National Science Foundation grant number in the Acknowledgements incorrectly read DMR-1420643; it should have read DMR-1420634. This has now been corrected in all versions of the Article.

Published online: 20 July 2020

https://doi.org/10.1038/s41563-020-0773-x

() The Author(s), under exclusive licence to Springer Nature Limited 2020

\section{Publisher Correction: Distinct handedness of spin wave across the compensation temperatures of ferrimagnets}

Changsoo Kim, Soogil Lee (iD, Hyun-Gyu Kim, Ji-Ho Park, Kyung-Woong Moon D, Jae Yeol Park (D), Jong Min Yuk, Kyung-Jin Lee (D), Byong-Guk Park (D), Se Kwon Kim (D), Kab-Jin Kim (D) and Chanyong Hwang (D)

Correction to: Nature Materials https://doi.org/10.1038/s41563-020-0722-8, published online 29 June 2020.

In the version of this Article originally published, Figs. 4 and 5 were mistakenly swapped; however, the captions were in the correct locations. This has now been corrected.

Published online: 2 September 2020

https://doi.org/10.1038/s41563-020-00813-3

(C) The Author(s), under exclusive licence to Springer Nature Limited 2020 